\title{
Clinical and cost effectiveness of mobile phone supported self monitoring of asthma: multicentre randomised controlled trial
}

\author{
(c) $\frac{(1)(8)}{\text { EY }}$ OPEN ACCESS
}

\author{
Dermot Ryan clinical research fellow ${ }^{1}$, David Price professor of primary care respiratory medicine ${ }^{1}$, \\ Stan D Musgrave research fellow ${ }^{1}$, Shweta Malhotra research assistant ${ }^{1}$, Amanda J Lee professor \\ of medical statistics ${ }^{2}$, Dolapo Ayansina research fellow ${ }^{2}$, Aziz Sheikh director of research ${ }^{3}$, Lionel \\ Tarassenko professor of electrical engineering, director ${ }^{4}$, Claudia Pagliari senior lecturer ${ }^{5}$, Hilary \\ Pinnock senior clinical research fellow ${ }^{3}$
}

${ }^{1}$ Academic Centre of Primary Care, Division of Applied Health Sciences, University of Aberdeen, Aberdeen AB25 2ZD, UK; ${ }^{2}$ Medical Statistics Team, Division of Applied Health Sciences, University of Aberdeen, Aberdeen; ${ }^{3}$ Allergy and Respiratory Research Group, Centre for Population Health Sciences, University of Edinburgh, Edinburgh EH8 9DX; ${ }^{4}$ Institute of Biomedical Engineering, University of Oxford, Oxford OX3 7DQ; ${ }^{5} \mathrm{eHealth}$ Research Group, Centre for Population Health Sciences, University of Edinburgh, Edinburgh

\begin{abstract}
Objective To determine whether mobile phone based monitoring improves asthma control compared with standard paper based monitoring strategies.
\end{abstract}

Design Multicentre randomised controlled trial with cost effectiveness analysis.

Setting UK primary care.

Participants 288 adolescents and adults with poorly controlled asthma (asthma control questionnaire (ACQ) score $\geq 1.5$ ) from 32 practices.

Intervention Participants were centrally randomised to twice daily recording and mobile phone based transmission of symptoms, drug use, and peak flow with immediate feedback prompting action according to an agreed plan or paper based monitoring.

Main outcome measures Changes in scores on asthma control questionnaire and self efficacy (knowledge, attitude, and self efficacy asthma questionnaire (KASE-AQ)) at six months after randomisation. Assessment of outcomes was blinded. Analysis was on an intention to treat basis.

Results There was no significant difference in the change in asthma control or self efficacy between the two groups (ACQ: mean change 0.75 in mobile group $v 0.73$ in paper group, mean difference in change -0.02 (95\% confidence interval -0.23 to 0.19$)$; KASE-AQ score: mean change $-4.4 v-2.4$, mean difference 2.0 (-0.3 to 4.2$)$ ). The numbers of patients who had acute exacerbations, steroid courses, and unscheduled consultations were similar in both groups, with similar healthcare costs. Overall, the mobile phone service was more expensive because of the expenses of telemonitoring.

Conclusions Mobile technology does not improve asthma control or increase self efficacy compared with paper based monitoring when both groups received clinical care to guidelines standards. The mobile technology was not cost effective.

Trial registration Clinical Trials NCT00512837.

\section{Introduction}

Globally, an estimated 300 million people have asthma, presenting a considerable and increasing burden of disease to healthcare systems, families, and patients. ${ }^{1}$ Despite two decades of asthma guidelines, ${ }^{2}$ asthma remains poorly controlled in a substantial proportion of people. ${ }^{3}$ Structured asthma management-which in the United Kingdom is predominantly delivered in primary care ${ }^{4}$ - can improve outcomes in terms of exacerbations, admissions to hospital, and days lost from school and work. ${ }^{5}$ The concept of supported self management, engaging both clinicians and patients in delivering and implementing regular monitoring of control and adjustment of treatment, is a key recommendation of national and international guidelines. ${ }^{67}$ The theoretical model developed by Glasziou and colleagues, using asthma as an exemplar, describes the complementary and evolving roles of periodic support from professionals and 
ongoing self monitoring by patients. ${ }^{8}$ Our recent qualitative study suggests that people with asthma perceive a role for mobile technology in aiding transition from clinician supported phases while control is gained to effective self management during maintenance phases. ${ }^{9}$

Poor adherence to monitoring and drugs is a potentially modifiable factor associated with poor control. ${ }^{10}$ In contrast with paper diary monitoring, in which as few as $6 \%$ of readings might be recorded, ${ }^{11}$ trials with electronic recording devices have shown rates of compliance of over $60 \%,{ }^{12}$ especially if the patient is aware that their health behaviour is being observed. ${ }^{13}$ Timely feedback of results to the patient can objectively show severity of symptoms and the impact of compliance with drug treatment. Mobile phones, the most pervasive and accessible form of technology globally, ${ }^{14}$ offer a highly convenient system for self monitoring coupled with instantaneous feedback, potentially engaging the patient in the monitoring and management of their asthma.

Set within the Medical Research Council's framework for the design and evaluation of complex interventions, ${ }^{15}{ }^{16}$ our phase III trial was underpinned by preliminary work that suggested that mobile phone based self monitoring of asthma care was feasible to deliver and acceptable to both patients and clinicians. ${ }^{912} 17$ We hypothesised that, in adolescents and adults with poorly controlled asthma offered treatment according to the British Thoracic Society/Scottish Intercollegiate Guideline Network (BTS-SIGN) asthma guideline, ${ }^{6}$ the use of mobile phone based monitoring of lung function and symptoms with feedback to patients would improve both control of asthma and patient self efficacy at six months compared with paper based monitoring strategies.

\section{Methods}

Our multicentre, investigator blinded randomised controlled trial was conducted in UK primary care in 2008-9. A detailed protocol has been published elsewhere ${ }^{18}$; we made no important changes to our methods during the trial.

\section{Practice recruitment}

The primary care research networks in Norfolk and Yarmouth, East Kent, North of England, and Essex and Hertfordshire identified and recruited practices.

\section{Participants}

We recruited patients aged 12 and over who were registered with participating practices, had poorly controlled asthma (defined as score $\geq 1.5$ on asthma control questionnaire (ACQ $)^{19}$ ), and had, or were willing to borrow, a compatible mobile phone handset and a contract with a compatible network. We excluded people who had other lung disease, were unable to communicate in English, or were receiving specialist care for severe/difficult asthma, and those whose general practitioner advised against inclusion for major social/clinical problems.

\section{Recruitment of patients}

We searched practice computer databases to identify potentially eligible patients. After the GP had screened the computer lists, we sent postal invitations to all eligible patients, with up to one written reminder.

One researcher (SDM or SM) prescreened respondents by telephone to determine whether they fulfilled the criterion of poorly controlled asthma and whether they had a contract with a compatible mobile phone network and a compatible handset.
Patients with an incompatible handset but who subscribed to a compatible network were offered the opportunity of borrowing a handset for the duration of the trial.

Potential participants attended a baseline assessment visit in their general practice at which they were provided with further information about the trial, eligibility was confirmed with the seven question version of the ACQ (which includes FEV (forced $^{\text {f }}$ expiratory volume in one second) in addition to the six questions described below as a primary outcome measure), and written consent was obtained. All consenting patients who met the eligibility criteria were enrolled into the trial.

\section{Primary outcome measures}

Asthma control questionnaire (ACQ)-The primary outcome measure was the change in asthma control between baseline and six months as measured by ACQ (six question version). ${ }^{2021}$ The ACQ measures clinical goals of asthma management on a scale of 0 (good control) to 6 (poor control), is responsive to change,$^{20}$ and has a minimum important difference within individuals of $0.5 .^{22}$ Postal administration gives comparable results with supervised completion. ${ }^{23}$

Knowledge, attitude, and self efficacy asthma questionnaire (KASE-AQ)-Self efficacy was measured as the change in KASE-AQ between the intervention and control groups at six months. The KASE-AQ has three independently scored subscales of 20 items of which we used two: "self efficacy," which measures perceived ability to control asthma, and "attitude to asthma." ${ }^{24}$ Scores range from 20 (minimum score) to 100 and are responsive to change. ${ }^{25} \mathrm{We}$ omitted the outdated knowledge subscale.

\section{Secondary outcome measures}

Full details of the secondary outcome measures of interest are in the published protocol. ${ }^{15}$ Briefly, we included:

- Mini-asthma quality of life questionnaire (mini-AQLQ): score from 1 (greatest impairment) to 7, with a minimum important difference of $0.5^{26}$

- Adverse occurrences obtained from the practice records, including admissions for exacerbations of asthma (defined as acute deteriorations of asthma for which the patient sought medical advice), prescribed courses of oral steroids, and unscheduled consultations

- Prescriptions of asthma drugs recorded in the patients' healthcare record

- Modified patient enablement instrument (mPEI): scale of 0 to 12 (most enabled) ${ }^{27}$

- Engagement with process (proportion of patients defaulting from clinical follow-up).

\section{Baseline assessment}

We undertook a baseline assessment comprising history of asthma, current smoking status, presence of comorbidity, and $\mathrm{FEV}_{1}$ with a Piko meter (nSpire Health, Enfield, UK). The use of the Piko meter to record peak flows was demonstrated and a meter given to the patient for use throughout the trial. The ACQ, KASE-AQ, mini-AQLQ, and mPEI questionnaires were self completed under supervision.

\section{Randomisation and protection against bias}

All consenting participants were stratified by practice and centrally randomised (Health Services Research Unit, University of Aberdeen) to mobile phone or paper based monitoring with 
a 1:1 allocation with random block sizes of two or four; telephone randomisation ensured concealment until the treatment was assigned. The practice nurse informed the patient of allocation to ensure the researchers were blinded to allocation throughout data collection and analysis.

\section{Mobile phone monitoring}

The practice nurse forwarded contact details of patients allocated to the intervention (mobile) group to OBS Medical (Abingdon, UK), who contacted the patient at a time convenient to them (normally within a week), downloaded the $t+$ Asthma application (fig $1 \Downarrow$ ) onto their (or a loaned) phone, tested its functionality, trained the patient in its use, provided details of web access, and followed up with a technical support call after one week of use.

The $\mathrm{t}+$ Asthma application enabled twice daily recording and transmission of symptoms, drug use, and peak flow. The mean of the five best peak flow values in the first 50 readings (after discarding the lowest five and highest five readings, which preliminary work suggested were often outliers ${ }^{28}$ ) was taken to be the target peak flow value $(100 \%)$ and used to define the $80 \%$ and $60 \%$ thresholds for a traffic light display. As new data were generated, the target peak flow value was adjusted to the mean of the five best peak flow values (after discarding outliers) in the most recent 50 readings. The recorded peak flow was displayed within the traffic light zones and the patient was prompted to follow their agreed action plan (fig $1 \Downarrow$ ). Incursion into the red or amber zones triggered contact by an asthma nurse from OBS Medical on the next working day. Appendix 1on bmj.com gives the protocol for these support calls. Both the patient and their clinician were able to access the patient data record via a password protected website.

\section{Paper based monitoring}

Patients in the control (paper) group were asked to keep a paper diary, recording the same data as the intervention group (symptoms, drug use, and peak flow readings) twice daily.

\section{Clinical care in both groups}

To ensure that our trial specifically tested the impact of the technology, we opted to provide the paper group with the same clinical care as the intervention group, rather than using (probably less intensive) usual care as a comparator. Before randomisation, the practice asthma nurse delivered a 30 minute standardised education session encompassing information on asthma, asthma treatment, inhaler technique, monitoring, and a personalised asthma action plan based on both symptoms and peak flows completed in accordance with the advice of the BTS-SIGN asthma guideline. Instruction on mobile or paper monitoring was provided after randomisation according to allocation.

Throughout the trial the practices' asthma nurse provided clinical care in accordance with the stepwise approach advocated by the BTS-SIGN asthma guideline. ${ }^{6}$ Patients were reviewed monthly (including the option of telephone reviews according to preference and clinical condition) until the nurse judged that control was achieved on the basis of clinical monitoring. Recognition of control in the context of a clinical review was discussed during the training. ${ }^{29}$ When control was attained, the patient was discharged from monthly follow-up but was encouraged to continue monitoring on a maintenance basis, seeking professional advice if needed.

\section{Data collection}

A researcher blinded to allocation collected primary outcome data at the final trial visit; non-attendees were sent the questionnaires by post. Interim data were collected by postal questionnaires at three months. The practice asthma nurses recorded the duration of each review at the end of the consultation and noted whether the patient's asthma was now controlled and whether a further appointment was needed. The researcher extracted data on adverse events (including admissions, unscheduled asthma consultations), asthma treatment categorised by BTS-SIGN guideline step, ${ }^{6}$ and use of healthcare resources over the six month trial period from the primary care records at baseline and at the end of the trial. Questionnaire data were scanned on the trial database. Data from records and other trial data were entered manually.

\section{Sample size calculations}

Using an estimated standard deviation of change in ACQ score of $0.25,{ }^{19}$ we estimated that a sample size of 125 per arm would have $90 \%$ power with a two sided $5 \%$ significance level to detect a difference in mean change in ACQ score of 0.1 or more between groups. With a standard deviation of $13.3,{ }^{25}$ a sample size of 39 in each arm would have $90 \%$ power with a two sided $5 \%$ significance level to detect a mean difference in mean KASE-AQ (self efficacy scale) score of 10 or more between groups.

\section{Data analysis}

Our main analysis was on an intention to treat (ITT) basis. We assumed that participants who did not attend the three or six month assessment had not improved their control and their previous results were therefore carried forward. ${ }^{30}$ A per protocol analysis was undertaken as a sensitivity analysis.

At baseline we described groups in terms of sociodemographic factors, history of asthma, ACQ, mini-AQLQ, KASE-AQ, and mPEI scores. We compared prespecified changes between groups from baseline in outcome measures using appropriate univariate techniques ( $t$ test, Mann-Whitney test). We used repeated measures analysis of variance, adjusted for age and sex, to examine trends over time in ACQ, mini-AQLQ, KASE-AQ, and mPEI scores both between and within groups. All two way interactions between outcome and age and sex were examined.

We compared the proportion of participants whose ACQ and mini-AQLQ score had improved by more than 0.5 (the minimum important difference),${ }^{31}$ the proportion in whom asthma remained poorly controlled (ACQ $>1.5^{19}$ ), and the proportion whose asthma was "well controlled" (ACQ $<0.75^{19}$ ) at six months.

We assessed costs from the perspective of the NHS. The total cost of the monitoring service for a projected maximum of 156 participants was allocated to each participant with the estimated reimbursed transmission costs. Costs were estimated by using unit costs obtained from standard sources ${ }^{32-34}$ (see table A in appendix 2 on bmj.com for details). We used $t$ tests to compare costs between groups.

All analyses were agreed a priori. We did not plan, or undertake, any interim analysis.

\section{Results}

\section{Recruitment}

Figure 2 shows the flow of patients through the trial $\Downarrow$. From 32 practices (total list size 311 926) computer searches identified 
a potentially eligible population of 13101 (mean (SD) age 48.2 (17.9): $63 \%$ female), of whom 1020 were excluded by their practice. A total of 12081 postal invitations were issued: 1016 people $(8.4 \%)$ expressed an interest in participating and were prescreened for eligibility (poor control and compatible mobile phone and network). The 393 potentially eligible patients were invited to attend the baseline assessment and 288 gave informed consent and were randomised: 145 to the mobile group and 143 to the paper group. Ten people had missing or incomplete ACQ scores at baseline and were excluded after randomisation, leaving 139 patients in each group for the intention to treat analysis.

\section{Baseline characteristics}

Demographic characteristics of participants, $\mathrm{FEV}_{1}$, smoking status, current asthma treatment, and baseline questionnaire scores were similar in both groups (table $1 \Downarrow$ ). Patients who did not complete the final questionnaires were older (51.7 (SD 17.7) $v 40.7$ (SD 17.3); $\mathrm{P}<0.001$ ) than patients who contributed to the final data collection, with no sex difference.

\section{Primary outcomes}

Asthma control-There was no significant difference in the change in asthma control in the two groups (ACQ: mean change 0.75 in mobile group $v 0.73$ in paper group, mean difference in change -0.02 ( $95 \%$ confidence interval -0.23 to 0.19 ) (table $2 \Downarrow$ ). Figure 3 shows changes over time $\Downarrow$. Over half the patients in both groups improved by more than the minimum important difference (table $3 \Downarrow$ ). At six months, 29/139 (21\%) of participants in the mobile group and $31 / 139$ (22\%) of the paper group had achieved the "well controlled" threshold of ACQ $\leq 0.75 .{ }^{19}$ Similarly, $69 / 139$ (50\%) of participants in the mobile group and $67 / 139(48 \%)$ of the paper group still scored above the "poorly controlled" threshold of ACQ $\geq 1.50 .{ }^{19}$

Self efficacy-The intention to treat analysis found no significant difference between the groups in change in the self efficacy and attitude scales of the KASE-AQ (table $2 \Downarrow$ ).

\section{Secondary outcomes}

Asthma related quality of life-Similarly, the intention to treat analysis showed no significant difference between the groups in change in mini-AQLQ score (table $2 \Downarrow$ ), though more patients in the mobile group improved their quality of life by more than the minimum important difference than in the paper group (table $3 \Downarrow)$.

Enablement-The intention to treat analysis found no significant difference between the groups in change in the mPEI scores (table $2 \Downarrow$ ).

\section{Per protocol sensitivity analysis}

The per protocol analysis included only data from participants who had complete information for each respective questionnaire at all time points. The results of this sensitivity analysis confirmed the findings of the intention to treat analysis (see table $\mathrm{B}$ in appendix 2 on bmj.com).

\section{Attendance for reviews, change in treatment, and unscheduled healthcare}

In both groups the median number of asthma consultations was two, with $37 \%$ of the patients in both groups attending only the baseline and one follow-up appointment (table $4 \Downarrow$ ). Most patients in both groups had increased treatment with asthma drugs according to the BTS-SIGN treatment steps $(82(59 \%)$ in the mobile group and 74 (55\%) in the paper group), and the nine patients not treated according to BTS-SIGN steps at baseline were receiving a recommended treatment schedule by the end of the trial (fig $4 \Downarrow$ ). There was no significant difference between the groups in the number of acute attacks, steroid courses prescribed, unscheduled GP or nurse consultations, out of hours visits, attendances at emergency department, or admissions (table $5 \Downarrow$ ).

\section{Trends over time}

Analysis of the repeat values over the three time points of the trial is shown in table $\mathrm{C}$ in appendix 2 on bmj.com. There was no significant difference in the improvement over time in any of the outcome measures.

\section{Cost of the monitoring services}

Table 6 gives details of the cost to the health service of providing the two monitoring services $\downarrow$. The only significant cost difference between the two groups was in relation to the telemonitoring service (about $£ 69$ ( $€ 83, \$ 108)$ per patient). The mean cost of providing respiratory care (including the nurse monitoring reviews) was $£ 246$ (SD £226) in the mobile group compared with $£ 245$ (SD $£ 201$ ) in the paper group (mean difference $-£ 1.26$ ( $-£ 51.47$ to $£ 48.95)$.

\section{Discussion}

\section{Summary of main findings}

The use of mobile phone technology did not significantly improve clinical outcomes, or increase self efficacy, compared with paper based monitoring when delivered in the context of guideline standard clinical care for the management of asthma. Healthcare costs were similar in both groups, with the telemonitoring expenses being an additional cost in the mobile group.

\section{Strengths and limitations}

We exceeded our estimated sample size for the intention to treat analysis, though not for the per protocol sensitivity analysis. The results, however, are consistent, and it is unlikely that a smaller attrition rate would alter our conclusions. Our a priori basis of carrying the previous result forward ${ }^{18}$ assumes that non-responders did not improve their control, which probably underestimates the degree of change in both groups.

The low recruitment rate $(2.4 \%$ of those invited) reflects both the low response rate ( $8.4 \%$ of those invited) and our requirement that participants should have poorly controlled asthma and a compatible mobile phone system. A slightly higher proportion of the participants was female, compared with the potentially eligible population. The 10 patients excluded after randomisation because of missing baseline ACQ scores were distributed between the two arms (four in the mobile group, six in the paper group), and were recruited from eight different practices, reducing the risk that this introduced important bias. Our multicentre trial was conducted in primary care, the setting for most asthma care in the UK, and included both an intention to treat analysis and a per protocol sensitivity analysis. We built on extensive formative work, which underpinned the hypothesis and informed the practical design of the intervention and the outcomes measured. ${ }^{9}{ }^{12} 1723$ Although the patients and nurses were clearly aware of the mode of monitoring that they were using, our trial procedures ensured that the researchers who undertook data collection and the trial statisticians were blinded to allocation (for example, randomisation was undertaken by 
the practice nurses, technical issues were dealt with by OBS Medical, patients were instructed not to reveal allocation, in the event of an inadvertent breach a second researcher attended the practice for subsequent data collection).

\section{Interpretation with reference to other research}

Our findings exemplify the challenges of evaluating complex eHealth interventions ${ }^{35}$ and the concern that potential benefits have thus far tended not to be substantiated by subsequent empirical evidence. ${ }^{36}$ Some studies using mobile phones ${ }^{37}$ and internet based monitoring have shown benefit in adults ${ }^{38}{ }^{39}$ and children ${ }^{40}$ when compared with "usual care." Usual care, however, is likely to be considerably less intensive than that experienced by intervention groups, so that it is often impossible to determine whether the observed benefits in these trials were because of the enhanced clinical care or the technological intervention. To overcome this, we provided the same structured clinical and educational intervention in both groups and showed that when the only difference is the mode of monitoring and associated feedback, the link with mobile phone technology had no significant effect.

Our hypothesis, building on formative work, ${ }^{9} 121317$ was predicated on the theory that using a mobile phone to monitor and receive instantaneous feedback on their asthma control would help patients to integrate management into everyday life, engage them more fully in their care, and thus potentially improve asthma control. Patients in both groups, however, attended a similar number of monthly clinical reviews, suggesting that the mobile phone monitoring did not improve compliance with care. Similarly, most patients in both groups stepped up their treatment during the course of the trial.

Participation in the trial probably resulted in greater engagement of patients and asthma nurses in both groups with "guideline standard care" (as opposed to "usual care"), potentially contributing to the improvement we observed in both groups. Spontaneous improvement over the timescale of the trial is another plausible explanation.

Despite the significant improvements in ACQ score in both groups, only about a fifth achieved the well controlled threshold of ACQ $=0.75$. While it has been shown that even better control can be achieved in trials with maximal doses of drugs in selected patients with asthma, ${ }^{41}$ the degree of improvement we observed is of the same order as that shown in "real life" trials, ${ }^{42}$ in which adherence is variable and comorbidity is common. To increase generalisability, we did not exclude smokers so some overlap with chronic obstructive pulmonary disease is possible.

The cost of the $\mathrm{t}+$ asthma service was additional to the health service costs, which were otherwise similar in both groups, making it the more expensive option. This might not extrapolate directly to normal clinical practice where, for example, economies of scale in a regional service could reduce the cost per patient. In addition, many other factors will affect both costs and benefits. Technological advances, such as smart phone applications, will increase functionality and widespread adoption might drive down costs. As part of the commercial arrangement with OBS Medical additional clinical support was available to the mobile group from the $\mathrm{t}+$ asthma nurse, though its contribution is unclear as it was not a closely monitored component of the trial.

A recent systematic review concluded that telehealthcare in asthma could have a role in reducing hospital admissions in high risk patients and in those with severe disease, ${ }^{43}$ and some studies have suggested that those with poorly controlled asthma might be more ready to comply with novel monitoring ${ }^{44}$ or take regular drug treatment ${ }^{45}$ than those with already well controlled asthma. Our trial, which recruited people with poorer control and lower baseline spirometry results than other trials, ${ }^{38}{ }^{39} \mathrm{did}$ not find a beneficial effect in this group.

\section{Conclusions and implications}

In people with poorly controlled asthma, the mobile phone based model of monitoring did not offer any advantages over and above paper based care when guideline standard clinical support services were provided to both groups. While mobile phone technology will appeal to some people, ${ }^{17}{ }^{18}$ it is not the crucial ingredient and carries cost implications. Its place in clinical care might depend on whether it is a cost effective option for enhancing "usual care" to the standards recommended by guidelines ${ }^{46}$ Policymakers internationally should consider the findings from this multicentre trial when considering the merits of investment in models of asthma care based on telehealthcare.

We thank the officers of the primary care research networks for supporting practice recruitment, the practices, practice nurses, and administrative staff for their active participation, and the patients who gave their time to participate in the trial. We thank Andrew Wilson and Neil Kendle for serving on the ITSC and Brian McKinstry and Chris Burton who offered advice as collaborators.

Contributors: DR initiated the idea for the study and with HP led the development of the protocol, securing of funding, study administration, data analysis, interpretation of results, and writing of the paper. DP, AS, $\mathrm{CP}$, and LT were grantholders who contributed to development of the protocol and securing of funding. DP, AS, and CP also contributed to study administration, data analysis, interpretation of results, and writing of the paper. LT contributed to interpretation of results and writing of the paper. SDM and SM undertook the data collection. AJL and DA were the trial statisticians. All authors had full access to all the data and were involved in interpretation of the data. DR and HP wrote the initial draft of the paper, to which all the authors contributed. DR and HP are study guarantors.

Funding: This study was funded by Asthma UK (project ID 07/047). Piko peak flow meters were donated by nSpire Health. HP is supported by a primary care research career award from the chief scientist's office of the Scottish Government.

Competing interests: All authors have completed the ICMJE uniform disclosure form at www.icmje.org/coi_disclosure.pdf (available on request from the corresponding author) and declare: DR has spoken on behalf of AstraZeneca, Nycomed, MSD, AlkAbello,Chiesi, Novartis, Pfizer and Boehringerlingelheim. He has undertaken advisory work for Astra Zeneca, MSD, Novartis, Uriach, Mundipharma, Orion, and Napp. DP has consultant arrangements with Boehringer Ingelheim,

GlaxoSmithKline, Merck, Mundipharma, Novartis, and Teva. In the past five years, he or his research team have received grants and support for research in respiratory disease from UK National Health Service, Aerocrine, AstraZeneca, Boehringer Ingelheim, GlaxoSmithKline, Merck, Mundipharma, Novartis, Nycomed, Pfizer, and Teva. He has spoken for AstraZeneca, Boehringer Ingelheim, Chiesi, GlaxoSmithKline, Merck, Mundipharma, Pfizer, and Teva. He has shares in AKL, which produces phytopharmaceuticals. He is the sole owner of Research in Real Life. SDM has received institutional grant support from Merck and Co, AKL Technologies, Chiesi, and Mundipharma AS serves on WHO's mHealth and Information Technology for Patient Safety Expert Working Groups and is an adviser to NHS Connecting for Health's Evaluation Programme. $\mathrm{He}$ is a consultant to ALK and Phadia and has received support from Napp, Pfizer, and Chiesi for research advice. LT is a cofounder of, advisor to, and holds shares in OBS Medical. He contributed to the design of $t+$ asthma and of the trial, but had no role in the day-to-day conduct of the study or statistical analysis. His work on telehealthcare is funded by the NIHR Biomedical Research Centre Programme. HP 


\section{What is already known on this topic}

Mobile phones offer a highly convenient system for self monitoring coupled with instantaneous feedback, potentially engaging patients in the monitoring and self management of their asthma

Some interventions using mobile phones in asthma care have shown benefit compared with "usual care," but it is often impossible to determine whether the observed benefits were because of the mobile phone intervention or the associated enhanced clinical care

\section{What this study adds}

The mobile phone based model of monitoring did not offer any clinical advantages over and above paper based care when guideline standard clinical support services were provided to both groups

Clinically relevant improvements were seen in both groups, suggesting that the telemonitoring was not the crucial ingredient in improving outcomes

The mobile phone based model of asthma care was more expensive than paper based model

has spoken for AstraZeneca, Boehringer Ingelheim, Chiesi,

GlaxoSmithKline, Pfizer, and Teva and undertaken advisory group work for Chiesi.

Ethical approval: This study was approved by Hertfordshire research ethics committee (07/H0311/126), and governance approval was provided by the participating NHS trusts.

Data sharing: No additional data available.

1 Masoli M, Fabian D, Holt S, Beasley R. The global burden of asthma. Global Initiative for Asthma, 2003. www.ginasthma.com.

2 Mclvor RA, Chapman KR. The coming of age of asthma guidelines. Lancet 2008;372:1021-2.

3 Rabe KF, Adachi M, Lai CK, Soriano JB, Vermeire PA, Weiss KB, et al. Worldwide severity and control of asthma in children and adults: the global asthma insights and reality surveys. $J$ Allergy Clin Immunol 2004;114:40-7.

4 Campbell S, Reeves D, Kontopantelis E, Middleton E, Sibbald B, Roland M. Quality of primary care in England with the introduction of pay for performance. N Engl J Med 2007;357:181-90

5 Gibson PG, Powell H, Wilson A, Abramson MJ, Haywood P, Bauman A, et al. Self-management education and regular practitioner review for adults with asthma. Cochrane Database Syst Rev 2002;3:CD001117.

6 British Thoracic Society/Scottish Intercollegiate Guideline Network. British Guideline on the Management of Asthma. Thorax 2008;63(suppl 4):1-121.

7 Global Initiative for Asthma (GINA). Global strategy for asthma management and prevention. 2010. www.ginasthma.org.

8 Glasziou P, Irwig L, Mant D. Monitoring in chronic disease: a rational approach. BMJ 2005;330:644-8.

9 Pinnock H, Slack R, Pagliari C, Price D, Sheikh A. Understanding the potential role of mobile phone-based monitoring on asthma self-management: qualitative study. Clin Exp Allergy 2007:37:794-802

10 Clatworthy J, Price D, Ryan D, Haughney J, Horne R. The value of self-report assessment of adherence, rhinitis and smoking in relation to asthma control. Prim Care Respir J 2009;18:300-5.

11 Yoos HL, Kitznian H, MeMullen A, Henderson C, Sidora K. Symptom monitoring in childhood asthma: a randomized clinical trial comparing peak expiratory flow rate with symptom monitoring. Ann Allergy Asthma Immunol 2002;88:283-91

12 Ryan D, Cobern W, Wheeler J, Price D, Tarassenko. Mobile phone technology in the management of asthma. J Telemed Telecare . 2005;11(suppl 1):43-6.

13 Reddel HK, Toelle BG, Marks GB, Ware SI, Jenkins CR, Woolcock AJ. Analysis of adherence to peak flow monitoring when recording of data is electronic. BMJ 2002;324:146-7.

14 International Telecommunication Union. Key global telecom indicators for the world telecommunication service sector.ITU, October 2010. www. itu.int.

15 Campbell NC, Murray E, Darbyshire J, Emery J, Farmer A, Griffiths F, et al. Designing and evaluating complex interventions to improve health care. BMJ 2007;334;455-9.

16 Craig P, Dieppe P, Maclntyre S, Mitchie S, Nazareth I, Petticrew M. Developing and evaluating complex interventions: new guidance. Medical Research Council, 2008.

17 Cleland J, Caldow J, Ryan D.Attitudes of patients and staff to using mobile phone technology to record and gather asthma data: a qualitative study. J Telemed Telecare 2007:13:85-9

18 Ryan D, Pinnock H, Lee AJ, Tarrasenko L, Pagliari C, Sheikh A, et al. The CYMPLA trial. Mobile phone-based structured intervention to achieve asthma control in patients with uncontrolled persistent asthma: a pragmatic randomised controlled trial. Prim Care Respir $J$ 2009;18:343-5.

19 Juniper E, Bousquet J, Abetz L, Bateman E. Identifying 'well-controlled' and not well controlled asthma using the Asthma Control Questionnaire. Respir Med 2006;100:616-21.

20 Juniper EF, O'Byrne PM, Guyatt GH, Ferrie PJ, King DR. Development and validation of a questionnaire to measure asthma control. Eur Resp J 1999;14:902-7.

21 Juniper EF, Svensson K, Mork Ann-Christin, Stahl E. Measurement properties and interpretation of three shortened versions of the asthma control questionnaire. Respir Med 2005;99:553-8.

22 Juniper EF, Ståhl E, O'Byrne PM. Minimal important difference for the asthma control questionnaire. Am J Respir Crit Care Med 2001;163:642.

23 Pinnock H, Sheikh A, Juniper E. Concordance between supervised and postal administration of the MiniAQLQ and ACQ is very high. J Clin Epidemiol 2005;58:809-14
24 Wigal JK, Stout C, Brandon M, Winder JA, McConnaughy K, Créer TL, et al. The knowledge, attitude, and self-efficacy asthma questionnaire. Chest 1993;104:1144-8.

25 Lemaigre V, Van den Bergh O, Van Hasselt K, De Peuter S, Victoir A, Verleden G. Understanding participation in an asthma self-management program. Chest 2005;128:3133-9.

26 Juniper EF, Guyatt GH, Cox FM, Ferrie PJ, King DR. Development and validation of the Mini Asthma Quality of Life Questionnaire. Eur Respir J 1999;14:32-8.

27 Howie JGR, Heaney DJ, Maxwell M, Walker JJ. A comparison of patient enablement instrument against two established satisfaction scales as an outcome measure of primary care consultations. Fam Pract 1998;15:165-71.

28 Cobern W. Asthma self-management using mobile telephone technology. www.ibme.ox. ac.uk/bsp/publications/pdf-files/Will\%20Cobern\%202007\%20DPhil\%20thesis.pdf.

29 Pinnock H, Fletcher M, Holmes S, Keeley D, Leyshon J, Price D, et al. Setting the standard for routine asthma consultations: a discussion of the aims, process and outcomes of reviewing people with asthma in primary care. Prim Care Respir J 2010;19:75-83.

30 White IR, Horton NJ, Carpenter J, Pocock SJ. Strategy for intention to treat analysis in randomised trials with missing outcome data. BMJ 2011;342:d40.

31 Guyatt GH, Juniper EF, Walter SD, Griffith LE, Goldstein RS. Interpreting treatment effects in randomised trials. BMJ 1998;316:690-3.

32 Curtis L. Unit costs of health and social care 2008. Personal Social Services Research Unit, University of Kent. www.pssru.ac.uk.

33 Department of Health. National schedule of reference costs 2007-08 for NHS Trusts and PCTs. www.dh.gov.uk/en/Publicationsandstatistics/Publications/ PublicationsPolicyAndGuidance/DH 098945.

34 British Medical Association and Royal Pharmaceutical Society. British National Formulary September 2008. BMJ Group and Pharmaceutical Press, 2008.

35 Catwell L, Sheikh A. Evaluating eHealth interventions: the need for continuous systemic evaluation. PLoS Med 2009;6(8):e1000126.

36 Black AD, Car J, Pagliari C, Anandan C, Cresswell K, BokunT, et al. The impact of eHealth on the quality and safety of health care: a systematic overview. PLOS Med 2011;8(1):e1000387.

37 Ostojic V, Cvoriscec B, Ostojic SB, Reznikoff D, Stipic-Markovic A, Tudjman Z. Improving asthma control through telemedicine: a study of short-message service. Telemed JE Health 2005;11:28-35.

38 Rasmussen LM, Phanareth K, Nolte $\mathrm{H}$, Backer V. Internet-based monitoring of asthma: a long-term, randomized clinical study of 300 asthmatic subjects. J Allergy Clin Immunol 2005:115:1137-42.

39 Van der Meer V, Bakker M, van den Hout WB, Rabe K, Sterk PJ, Kievit J, et al. Internet-based self-management plus education compared with usual care in asthma a randomized trial. Ann Intern Med 2009;151:110-20.

40 Jan R-L, Wang J-Y, Huang M-C, Tseng S-M, Su H-J, Liu L-F. An internet-based interactive telemonitoring system for improving childhood asthma outcomes in Taiwan. Telemed $J$ E Health 2007;13:257-68.

41 Bateman ED, Boushey HA, Bousquet J, Busse WW, Clark TJH, Pauwels RA, et al. Can guideline-defined asthma control be achieved? Am J Respir Crit Care Med 2002;170:836-44

42 Price DB, Musgrave SD, Shepstone L, Hillyer EV, Sims EJ, Gilbert RFT, et al. Leukotriene antagonists as first-line or add-on asthma-controller therapy. N Eng J Med 2011;364:1695-707.

43 McLean S, Chandler D, Nurmatov U, Liu J, Pagliari C, Car J, et al. Telehealthcare for asthma. Cochrane Database Syst Rev 2010;10:CD007717

44 Van der Meer V, van Stel HF, Detmar SB, Otten W, Sterk PJ, Sont JK. Internet-based self-management offers an opportunity to achieve better asthma control in adolescents. Chest 2007;132;112-9.

45 Liu WT, Huang CD, Wang CH, Lee KY, Lin SM, Kuo HP. A mobile-telephone based interactive self care system improves asthma control. Eur Resp J 2011;37:310-7.

46 McLean S, Protti D, Sheikh A. Telehealthcare for long term conditions. BMJ 2011;342:d120.

Accepted: 13 January 2012

\section{Cite this as: BMJ 2012:344:e1756}

This is an open-access article distributed under the terms of the Creative Commons Attribution Non-commercial License, which permits use, distribution, and reproduction in any medium, provided the original work is properly cited, the use is non commercial and is otherwise in compliance with the license. See: http://creativecommons.org/licenses/by$\mathrm{nc} / 2.0 /$ and http://creativecommons.org/licenses/by-nc/2.0/legalcode. 


\section{Tables}

Table 1| Baseline characteristics of people with asthma allocated to mobile phone or paper based monitoring. Figures are numbers (percentages) unless stated otherwise

\begin{tabular}{lcc} 
& Mobile (n=145) & Paper (n=143) \\
Mean (SD) age (years) & $46.6(18.0)$ & $51.5(17.7)$ \\
\hline Female & $96(66)$ & $84(59)$ \\
\hline White ethnicity & $141(97)$ & $142(99)$ \\
\hline Median (IQR) FEV ${ }_{1}$ percentage predicted ${ }^{\star}$ & $83.1(71.0-96.6)$ & $80.8(65.3-94.9)$ \\
\hline Smoking statust & & $72(50)$ \\
\hline Never smoked & $70(49)$ & $54(38)$ \\
\hline Ex-smoker & $55(38)$ & $16(11)$ \\
\hline Current smoker & $19(13)$ & $7(5)$ \\
\hline Treatment step: & & $33(24)$ \\
\hline Step 0: no current drugs & $8(6)$ & $41(30)$ \\
\hline Step 1: short acting $\beta_{2}$ agonist & $41(29)$ & $53(39)$ \\
\hline Step 2: inhaled steroid & $47(33)$ & $3(2)$ \\
\hline Step 3: long acting $\beta_{2}$ agonist or leukotriene receptor antagonist & $42(30)$ & $6(4)$ \\
\hline Step 4: high dose steroids or additional treatment & $4(3)$ & $2.29(0.77)$ \\
\hline Treatment not in line with guideline treatment steps & $3(2)$ & $78.0(10.5)$ \\
\hline Mean (SD) score on questionnaires: & & $79.4(7.2)$ \\
\hline ACQ & $2.32(0.73)$ & $4.34(1.08)$ \\
\hline KASE-AQ (self efficacy domain) & $77.4(9.5)$ & $6.93(3.78)$ \\
\hline KASE-AQ (attitude domain) & $80.0(7.4)$ & \\
\hline mini-AQLQ & $4.25(0.91)$ & $5.83(3.67)$ \\
\hline mPEI & & \\
\hline
\end{tabular}

$I Q R=$ interquartile range; $A C Q=$ asthma control questionnaire; $K A S E-A Q=k n o w l e d g e$, attitude, and self efficacy asthma questionnaire; $A Q L Q=a s t h m a$ quality of life questionnaire; $\mathrm{mPEI}=$ modified patient enablement instrument.

${ }^{*}$ Available for 142 in mobile group and 140 in paper group.

†Available for 144 in mobile group and 142 in paper group. 
Table 2| Intention to treat analysis of asthma control and self efficacy in people with asthma randomised to mobile phone or paper based monitoring

\begin{tabular}{|c|c|c|c|c|c|}
\hline & \multirow[b]{2}{*}{ No in group } & \multicolumn{2}{|c|}{ Mean (SD) } & \multirow[b]{2}{*}{ Mean change $(95 \% \mathrm{Cl})$} & \multirow{2}{*}{$\begin{array}{l}\text { Mean difference of } \\
\text { mean change ( } 95 \% \\
\mathrm{Cl})\end{array}$} \\
\hline & & Baseline & 6 months & & \\
\hline \multicolumn{6}{|c|}{ Primary outcomes } \\
\hline \multicolumn{6}{|l|}{ ACQ: } \\
\hline Mobile & 139 & $2.32(0.73)$ & $1.57(0.99)$ & 0.75 (0.61 to 0.89$)$ & \multirow[t]{2}{*}{$-0.02(-0.23$ to 0.19$)$} \\
\hline Paper & 139 & $2.29(0.77)$ & $1.56(1.09)$ & 0.73 (0.57 to 0.89$)$ & \\
\hline \multicolumn{6}{|c|}{ KASE-AQ (self efficacy scale): } \\
\hline Mobile & 110 & $77.4(9.5)$ & $81.8(11.1)$ & $-4.4(-6.1$ to -2.7$)$ & \multirow[t]{2}{*}{$2.0(-0.3$ to 4.2$)$} \\
\hline Paper & 121 & $78.0(10.5)$ & $80.4(11.5)$ & $-2.4(-3.9$ to -0.9$)$ & \\
\hline \multicolumn{6}{|c|}{ KASE-AQ (attitude scale): } \\
\hline Mobile & 118 & $80.0(7.4)$ & $81.8(9.5)$ & $-1.7(-2.9$ to -0.6$)$ & \multirow[t]{2}{*}{$-0.2(-1.6$ to 1.6$)$} \\
\hline Paper & 122 & $79.4(7.2)$ & $81.2(8.6)$ & $-1.8(-2.9$ to -0.6$)$ & \\
\hline \multicolumn{6}{|c|}{ Questionnaire based secondary outcomes } \\
\hline \multicolumn{6}{|c|}{ mini-AQLQ: } \\
\hline Mobile & 97 & $4.25(0.91)$ & $5.0(1.32)$ & $-0.75(-0.94$ to -0.57$)$ & \multirow[t]{2}{*}{$0.10(-0.16$ to 0.34$)$} \\
\hline Paper & 104 & $4.34(1.08)$ & $4.99(1.34)$ & $-0.65(-0.84$ to -0.46$)$ & \\
\hline \multicolumn{6}{|l|}{ mPEl: } \\
\hline Mobile & 136 & $5.83(3.67)$ & $6.79(4.0)$ & $-0.96(-1.62$ to -0.31$)$ & \multirow[t]{2}{*}{$1.19(0.26$ to 2.11$)$} \\
\hline Paper & 35 & $6.93(3.78)$ & $6.71(3.92)$ & $0.22(-0.44$ to 0.88$)$ & \\
\hline
\end{tabular}

$\mathrm{ACQ}=$ asthma control questionnaire; $\mathrm{KASE}-\mathrm{AQ}=$ knowledge, attitude, and self efficacy asthma questionnaire; $\mathrm{AQLQ}=$ asthma quality of life questionnaire; $\mathrm{mPEI}=$ modified patient enablement instrument. 
Table 3| Proportion of people with asthma whose ACQ and mini-AQLQ score improved by more than minimum important difference (MID) at six months according to randomisation to mobile phone or paper based monitoring

\begin{tabular}{|c|c|c|c|c|c|c|}
\hline & \multirow[b]{2}{*}{ No in group } & \multicolumn{2}{|c|}{ Improvement } & \multicolumn{2}{|c|}{ Deterioration } & \multirow[b]{2}{*}{ Between group $P$ value } \\
\hline & & $\geq$ MID & $<$ MID & $<$ MID & $\geq$ MID & \\
\hline \multicolumn{7}{|l|}{$A C Q$} \\
\hline Mobile & 139 & $83(60)$ & $44(32)$ & $6(4)$ & $6(4)$ & \multirow[t]{2}{*}{0.78} \\
\hline Paper & 139 & $79(57)$ & $44(32)$ & $6(4)$ & $10(7)$ & \\
\hline \multicolumn{7}{|c|}{ mini-AQLQ } \\
\hline Mobile & 97 & $43(80)$ & $3(6)$ & $4(7)$ & $4(7)$ & \multirow[t]{2}{*}{0.03} \\
\hline Paper & 104 & $39(58)$ & $16(24)$ & $5(8)$ & $7(10)$ & \\
\hline
\end{tabular}

$A C Q=a s t h m a$ control questionnaire; $A Q L Q=a s t h m a$ quality of life questionnaire. 
Table 4 | Use of healthcare resources during follow-up in people with asthma allocated to mobile phone or paper based monitoring

\begin{tabular}{lcc} 
& Mobile (n=140) & Paper (n=141) \\
Mean (SD) asthma consultations during trial ${ }^{*}$ & $2.4(1.2)$ & $2.7(1.3)$ \\
\hline No attending follow-up consultations: & & \\
\hline Baseline clinical consultation only & $37(26 \%)$ & $27(19 \%)$ \\
\hline Baseline + 1 follow up & 52 & 51 \\
\hline Baseline + 2 follow-ups & 28 & 28 \\
\hline Baseline + 3 follow-ups & 13 & 16 \\
\hline Baseline + 4 follow-ups & 3 & 9 \\
\hline Baseline + 5 follow-ups & 5 & 10 \\
\hline Baseline + 6 follow-ups & 0 & 0 \\
\hline Total No of follow-up consultations & 184 & 241 \\
\hline Mode of consultation: & 173 & 231 \\
\hline Face to face & 11 & 10 \\
\hline Telephone & & \\
\hline No who changed BTS-SIGN treatment step: & $82(59 \%)$ & $74(55 \%)$ \\
\hline Stepped up & $50(36 \%)$ & $50(35 \%)$ \\
\hline Step unchanged & $11(8 \%)$ & $13(9 \%)$ \\
\hline Stepped down & $3(2 \%)$ & $6(4 \%)$ \\
\hline Brought into line with guideline treatment steps & & \\
\hline
\end{tabular}

*Mean difference 0.3 (95\% confidence interval -0.03 to 0.6$), P=0.07$.

$\dagger P=0.46$. 
Table 5 | Details of unscheduled care and acute exacerbations during follow-up in people with asthma allocated to mobile phone or paper based monitoring

Mobile $n=140$ Paper $n=141 \quad P$ value

Consultations with GP for asthma

\begin{tabular}{|c|c|c|c|}
\hline Total No of consultations & 108 & 85 & - \\
\hline No (\%) with at least one & $51(36)$ & $41(29)$ & - \\
\hline Median (IQR) & $0(0-1)$ & $0(0-1)$ & 0.19 \\
\hline \multicolumn{4}{|c|}{ Unscheduled general practice nurse consultations: } \\
\hline Total No of consultations & 86 & 109 & - \\
\hline No (\%) with at least one & $45(32)$ & $62(44)$ & - \\
\hline Median (IQR) & $0(0-1)$ & $0(0-1)$ & 0.07 \\
\hline \multicolumn{4}{|l|}{ Out of hours attendances: } \\
\hline Total No of attendances & 3 & 5 & - \\
\hline No (\%) with at least one & $3(2)$ & $5(4)$ & - \\
\hline Median (IQR) & $0(0-0)$ & $0(0-0)$ & 0.70 \\
\hline \multicolumn{4}{|c|}{ Emergency department attendances: } \\
\hline Total No of attendances & 3 & 0 & - \\
\hline No (\%) with at least one & $3(2)$ & 0 & - \\
\hline Median (IQR) & $0(0-0)$ & $0(0-0)$ & 0.08 \\
\hline \multicolumn{4}{|l|}{ Admissions for asthma: } \\
\hline Total No of admissions & 3 & 1 & - \\
\hline No (\%) with at least one & $3(2)$ & $1(1)$ & - \\
\hline Median (IQR) & $0(0-0)$ & $0(0-0)$ & 0.32 \\
\hline \multicolumn{4}{|l|}{ Acute exacerbations: } \\
\hline Total No of exacerbations & 74 & 70 & - \\
\hline No (\%) with at least one & $43(31)$ & $45(32)$ & - \\
\hline Median (IQR) & $0(0-1)$ & $0(0-1)$ & 0.84 \\
\hline \multicolumn{4}{|l|}{ Steroid courses: } \\
\hline Total No of steroid courses & 39 & 43 & 一 \\
\hline No (\%) with at least one & $28(20)$ & $30(21)$ & - \\
\hline Median (IQR) & $0(0-0)$ & $0(0-0)$ & 0.79 \\
\hline
\end{tabular}




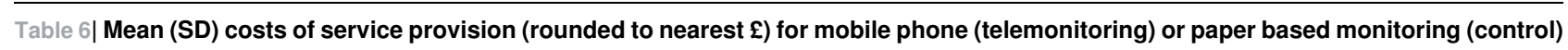

\begin{tabular}{lcccc} 
& Telemonitoring ( $\mathbf{n}=\mathbf{1 4 0})$ & Control $(\mathbf{n}=\mathbf{1 4 1})$ & Mean difference (95\% Cl) $\mathbf{P}$ value \\
\hline Total healthcare costs & $315(226)$ & $245(201)$ & $-70(-121$ to -20$)$ & 0.006 \\
\hline Total cost of delivering trial interventions according to allocation & $94(12)$ & $28(12)$ & $-66(-69$ to -64$)$ & $<0.001$ \\
\hline Trial nursing costs & $25(12)$ & $28(12)$ & $3(0$ to 6$)$ & 0.07 \\
\hline Telemonitoring service costs & 69 & 0 & - & - \\
\hline Total cost of healthcare provision (excluding trial interventions) & $53(143)$ & $41(87)$ & $-12(-40$ to 16$)$ & 0.39 \\
\hline GP respiratory consultations & $26(47)$ & $21(42)$ & $-6(-16$ to 5$)$ & 0.30 \\
\hline Practice nurse respiratory consultations & $6(12)$ & $8(12)$ & $2(-1$ to 4) & 0.26 \\
\hline Secondary care costs (outpatient and admissions) & $18(116)$ & $11(56)$ & $-7(-29$ to 14$)$ & 0.51 \\
\hline Emergency services (including emergency department, out of hours) & $3(17)$ & $2(13)$ & $-1(-4$ to 3) & 0.61 \\
\hline Total cost of prescriptions for respiratory drugs & $167(136)$ & $178(152)$ & $11(-23$ to 45) & 0.53 \\
\hline
\end{tabular}




\section{Figures}

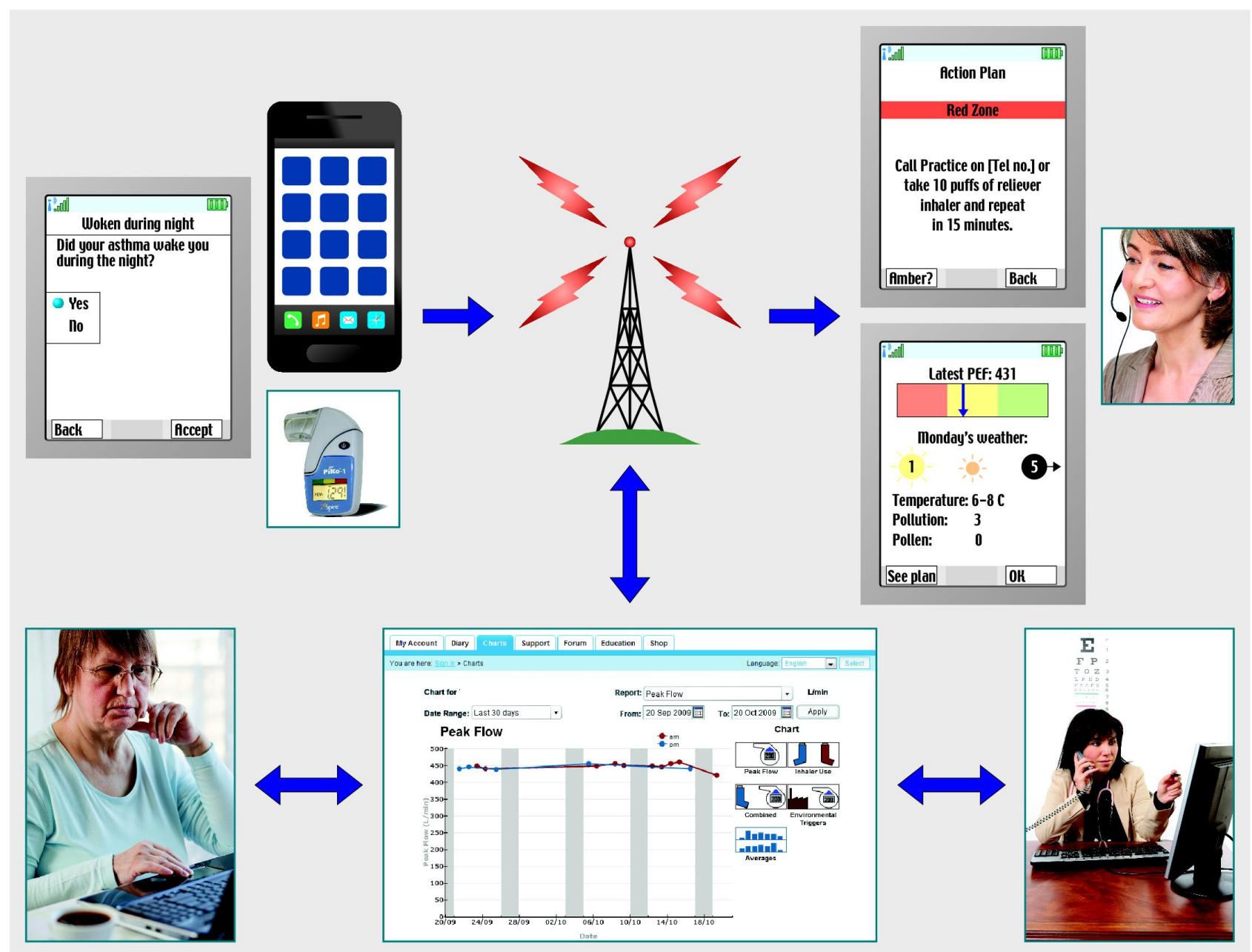

Fig 1 Mobile phone monitoring system. $t+$ Asthma application, running on web enabled handset, allowed patient to record symptoms, drug use, and peak flow from Piko meter. Automated display plotted peak flow as normal ( $\geq 80 \%$ green zone), in need of attention (60-79\% amber zone), or in need of urgent action ( $<60 \%$ red zone) and prompted patients to follow their agreed action plan. Incursion into red or amber zones also triggered contact by $t+$ Asthma nurse from OBS Medical on next working day to ascertain what had happened and what learning points had arisen. Data were automatically transmitted to secure website on remote server hosted by OBS Medical, every time application was used by patient. Patient and clinician were able to access patient data record via password protected website, which also provided general information about asthma 


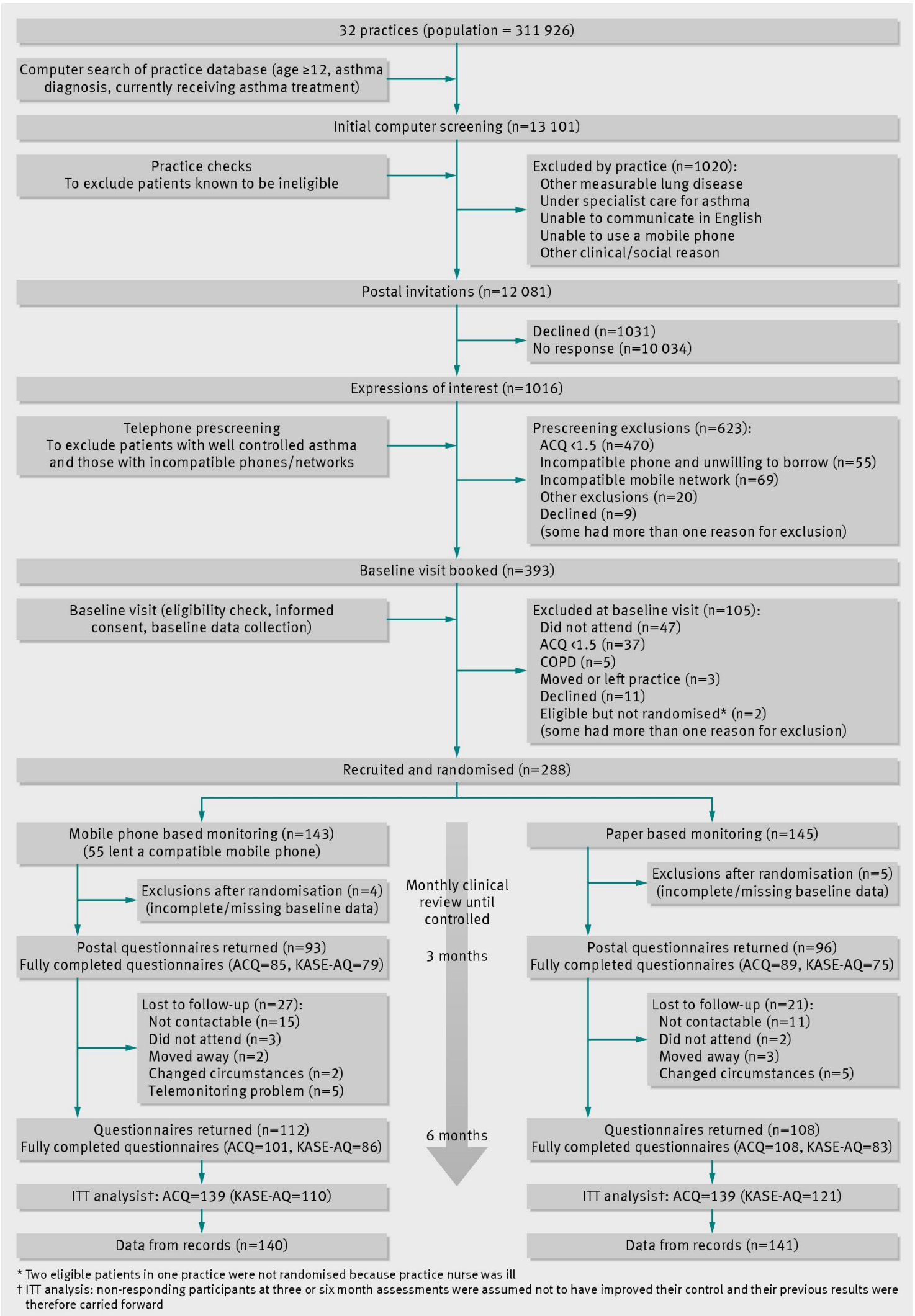

Fig 2 Flow of patients through trial of mobile phone and paper based monitoring of asthma control $(A C Q=a s t h m a$ control

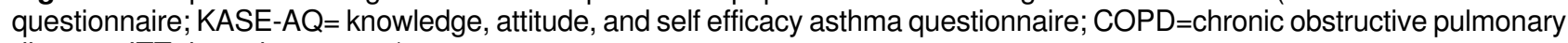
disease; ITT=intention to treat) 


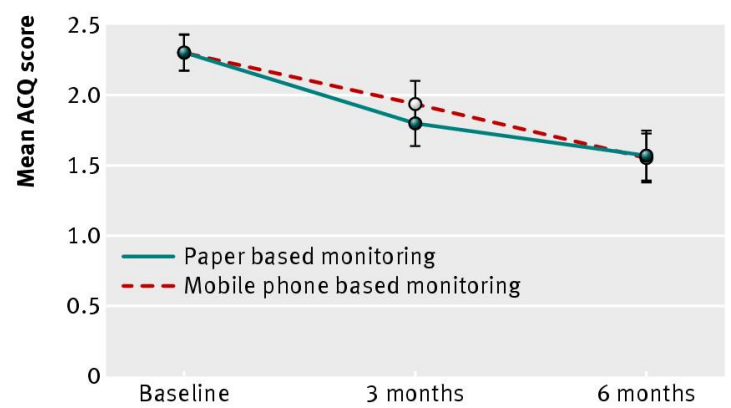

Fig 3 Mean ACQ scores adjusted for age and sex at different time points in people with asthma according to allocated method of monitoring

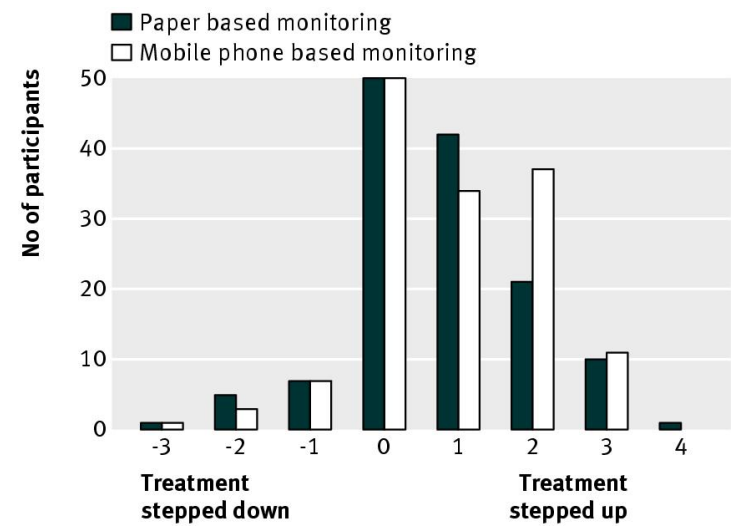

Fig 4 Change in BTS-SIGN step from baseline to six months in people with asthma according to allocated method of monitoring 